\title{
Le Sillon Lorrain : quelle recomposition territoriale dans un espace multipolaire?
}

Julien Gingembre

\section{OpenEdition}

\section{Journals}

Édition électronique

URL : https://journals.openedition.org/cdg/1375

DOI : $10.4000 /$ cdg. 1375

ISSN : 2107-7266

Éditeur

UMR 245 - CESSMA

Référence électronique

Julien Gingembre, « Le Sillon Lorrain : quelle recomposition territoriale dans un espace multipolaire? », Carnets de géographes [En ligne], 11 | 2018, mis en ligne le 15 septembre 2018, consulté le 20 mai 2021. URL : http://journals.openedition.org/cdg/1375; DOI : https://doi.org/10.4000/cdg.1375

Ce document a été généré automatiquement le 20 mai 2021.

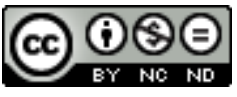

La revue Carnets de géographes est mise à disposition selon les termes de la Licence Creative Commons Attribution - Pas d'Utilisation Commerciale - Pas de Modification 4.0 International. 


\title{
Le Sillon Lorrain : quelle recomposition territoriale dans un espace multipolaire?
}

\author{
Julien Gingembre
}

\section{Introduction}

$1 \mathrm{Au}$ lendemain d'une réforme territoriale qui a profondément transformé la carte régionale, la question métropolitaine demeure plus que jamais centrale dans le débat politique. Avec la poursuite de la décentralisation, la France renforce le pouvoir de ses villes en leur fournissant des outils de gouvernance plus intégrés afin de mieux s'insérer dans la compétition interurbaine qui se joue à l'échelle européenne. Récemment, deux outils ont été créés. Issues de la loi MAPTAM du 27 janvier 2014, les métropoles en sont un des derniers aboutissements. À leurs côtés, les pôles métropolitains, créés plut tôt, dès 2010 avec la Réforme des collectivités territoriales, ont connu un vif engouement de la part des élus urbains au moment de leur création (Béhar et alii, 2011).

2 En Lorraine, la configuration géographique singulière de l'armature urbaine, avec la proximité de Nancy et de Metz et l'importance de son axe urbain central, le Sillon Lorrain, a été à l'origine d'une recomposition territoriale profonde et multiscalaire. Le pôle métropolitain du Sillon Lorrain, créé en 2011, regroupe les intercommunalités d'Épinal, de Nancy, de Metz et de Thionville. De création récente, le pôle s'appuie néanmoins sur l'ancienneté du rapprochement de Nancy et de Metz dans le cadre de la métropole d'équilibre. Plus récemment, la communauté urbaine du Grand Nancy et la communauté d'agglomération de Metz ont obtenu le droit de se transformer en métropole. À moyen terme, Metz et Thionville envisagent une fusion de leurs intercommunalités. Dans tous les cas, il s'agit toujours de «peser plus dans le débat régional » et de « s'imposer entre Paris et Strasbourg »" . 
3 Cet espace original par sa configuration et ses enjeux économiques demeure assez peu exploré par la recherche. La géographie régionale lui a naturellement préféré l'étude de la Lorraine dans son ensemble (Frécaut, 1983). Bien entendu, le Sillon Lorrain, en tant qu'axe majeur de développement, y tient une place importante. Mais l'étude de cet espace dans une perspective idiographique fait globalement défaut. La refonte de la carte régionale pose pourtant la question de la pertinence du nouvel ensemble dans lequel s'inscrit la Lorraine ${ }^{2}$ (Woessner, 2004). Ainsi, il paraît essentiel se pencher sur cet espace métropolitain émergent. Au-delà de son caractère frontalier, qui est fondamental, le Sillon est un objet géographique riche qui questionne les concepts de métropolisation, de réseau de villes et encore de polycentrisme.

À travers la prolongation de réflexions soulevées lors d'une recherche doctorale menée en collaboration CIFRE avec l'Agence d'urbanisme de Nancy et portant sur l'émergence $\mathrm{du}$ pôle métropolitain du Sillon Lorrain, l'objectif de l'article est de montrer le caractère singulier de la construction métropolitaine en Lorraine. Pour cela, l'article propose une analyse rétrospective analysant la construction politique dans le temps long et s'appuyant sur le concept de recomposition territoriale. L'article revient sur le contexte de la naissance de la conscience régionale en Lorraine, puis sur l'émergence d'une vision métropolitaine au sein du couple Metz-Nancy. Ces éléments orientent fortement la construction métropolitaine actuelle. L'article analyse ensuite les évolutions récentes du Sillon Lorrain et interroge son devenir. Mais d'abord, afin de comprendre les enjeux actuels, nous présentons l'espace étudié ainsi que la démarche méthodologique mobilisée.

\section{Cadres géographique et méthodologique}

\subsection{Le Sillon Lorrain : un espace difficile à délimiter}

Espace politique émergent mais déjà bien installé dans le paysage institutionnel du Grand Est, le pôle métropolitain du Sillon Lorrain s'inscrit toutefois un ensemble spatial difficile à délimiter. Cet espace est surtout assimilé au couple Nancy-Metz, mais il regroupe également les intercommunalités d'Épinal et de Thionville. Si elles ne sont pas des métropoles - au sens fonctionnel - à part entière, Nancy et Metz ne sont pas non plus considérées comme des villes moyennes : elles regroupent chacune plus de 100000 habitants dans leur commune et plus de 200000 habitants dans leur unité urbaine. Mais ce statut « intermédiaire » rend leur affichage plus flou. En effet, les plus grandes villes sont plus médiatisées (notamment depuis la loi MAPTAM et avec les nombreux classements des métropoles) tandis que les villes de l'échelon inférieur, dites "moyennes ", ont récemment connu un regain d'intérêt des chercheurs ${ }^{3}$ et de l'État, avec comme dernière actualité la sélection par le Gouvernement de 222 villes moyennes qui bénéficieront d'un accompagnement financier afin de revitaliser leur centre-ville*

6 En 2013, les quatre établissements publics composant le pôle métropolitain regroupaient près de 634000 habitants. C'est moins que l'addition des quatre agglomérations (au sens de l'unité urbaine de l'INSEE, soit 765000 habitants), mais cette donnée est plus proche de la réalité fonctionnelle de cet espace discontinu. Enfin, élargi aux aires urbaines, le total est porté à 1053000 habitants. Dans tous les cas, Metz et Nancy représentent au moins les trois-quarts du total. 
7 Les quatre villes du Sillon, en particulier Metz et Nancy, polarisent un vaste espace alentour, ce qui est notamment permis par un réseau de transport ferré dense et des voies autoroutières encore gratuites. Si les quatre aires urbaines du Sillon ne sont pas toutes contiguës, leurs marges sont toutefois très proches.

8 Mais cette gymnastique démographique interroge alors l'intégration dans le décompte des espaces interstitiels comme Pont-à-Mousson, agglomération de 24000 habitants située à mi-distance entre Metz et Nancy, ou encore les espaces en marge du Sillon mais polarisés par celui-ci : c'est notamment le cas de Toul et Lunéville à proximité de Nancy, ou bien encore de Briey et de Jarny à l'ouest de Metz.

9 Le Sillon Lorrain, clairement défini dans le pôle métropolitain, demeure ainsi un objet à géographie variable quand il est étudié comme un espace. Les habitants et leurs multiples pratiques ne font pas encore émerger un espace vécu multipolaire. Mais les flux de mobilité domicile-travail entre Nancy et Metz s'imposent comme l'un des éléments fonctionnels les plus structurants pour le pôle. Parmi ces navetteurs lorrains, les cadres tiennent une place centrale dans le processus de métropolisation (Gingembre et Baude, 2014). Mais la discontinuité spatiale demeure un frein important, particulièrement entre Épinal, Nancy et Metz où les distances sont grandes $(56 \mathrm{~km}$ entre Nancy et Metz; $73 \mathrm{~km}$ entre Épinal et Nancy). Ce n'est pas le cas entre Metz et Thionville où la distance est plus réduite. Les deux agglomérations forment un ensemble quasi continu, sorte de conurbation construite sur les liens étroits tissés notamment à l'époque de l'essor de la sidérurgie.

10 La coalescence des agglomérations de Metz et de Nancy, qui fut prophétisée par les travaux de l'ORÉAM Lorraine (Organisme Régional d'Étude et d'Aménagement d'Aire Métropolitaine) au moment de la définition de la métropole d'équilibre, n'a pas eu lieu. La faute à un important ralentissement démographique initié dès les débuts des crises industrielles, qui a parfois même signifié perte de population, sans toutefois atteindre les niveaux des « villes rétrécissantes » allemandes ou américaines.

11 Aujourd'hui, le pôle métropolitain tente un difficile rapprochement entre quatre entités dont les enjeux et les ambitions sont individuels. Les difficultés rencontrées aujourd'hui furent pourtant déjà rencontrées dans les années 1960 avec la première expérience métropolitaine portée en Lorraine. Afin d'étudier cela de manière rétrospective, nous nous appuyons sur le concept de recomposition territoriale.

\subsection{Démarche et méthode de la géographie appliquée}

Les questionnements récents sur la métropolisation et ses conséquences ne sont pas l'exclusivité du champ académique. En effet, l'État s'est intéressé à la question métropolitaine et aux bouleversements engendrés par la métropolisation, notamment par l'intermédiaire de la DATAR. D'autre part, aux échelons inférieurs, les collectivités s'accaparent également le sujet. Souvent, c'est aux agences d'urbanisme et de développement que sont confiées ces missions d'analyse et de prospective. Les agences d'urbanisme sont des instruments d'ingénierie publique mutualisée. Leur rôle est d'aider les élus des collectivités membres à fixer et mettre en œuvre leurs politiques d'aménagement et de développement territorial et urbain.

13 Fruit d'une hybridation entre recherche et action urbaine permise par une collaboration CIFRE, ce travail s'est appuyé sur une démarche de recherche appliquée 
combinant analyse spatiale et observation participative de terrain. Pour Roger Brunet et alii (1993 : 37), la géographie appliquée est « un ensemble d'études et de recherches géographiques orientées par ou vers la demande d'institutions publiques ou d'entreprises privées, et relevant de l'aménagement du territoire ou de la stratégie des localisations et de l'environnement». Elle constitue ici un pont entre la sphère universitaire et celle de l'aménagement du territoire.

Cette recherche s'appuie sur l'observation et l'exploration des débats portés par les élus du Sillon Lorrain et des acteurs socio-économiques lors des conférences publiques et réunions de travail organisées au moment où émergeait le pôle métropolitain. Ainsi, à l'issue de la création du pôle en décembre 2011, une première conférence fut organisée le 5 juillet 2012 à Pont-à-Mousson. Cet événement fut suivi d'autres rencontres, organisées dans le cadre de la Conférence métropolitaine, émanation de l'ancienne association du Sillon Lorrain. En réunissant acteurs politiques, décideurs, techniciens mais aussi chercheurs, ces conférences, débats et tables-rondes ont permis de faire ressortir les principales préoccupations et interrogations liées à la naissance et à la construction du pôle.

Ce matériau de recherche vient compléter le corpus bibliographique sur le sujet. Il se compose d'une part de monographies régionales telles que Géographie de la Lorraine (Frécaut (dir.), 1983), Histoire administrative de la Lorraine (Masson, 1982) ou la thèse de Brunot Moriset (1995), et d'autre part des documents techniques (notamment les documents d'aménagement) édités par l'ORÉAM Lorraine, par la DATAR et, plus récemment, par les collectivités et les agences d'urbanisme lorraines ainsi que par le pôle métropolitain lui-même.

16 Ces sources, par leur relative ancienneté (les plus anciennes sont contemporaines de la métropole d'équilibre des années 1960) permettent un regard à la fois rétrospectif sur l'émergence de la question métropolitaine en Lorraine et comparatif dans le sens où il nous est possible de confronter les politiques menées sur ce sujet depuis un demi siècle.

\subsection{La recomposition territoriale : une clé de lecture pour analyser la construction politique du Sillon Lorrain}

17 Le concept de recomposition territoriale permet de comprendre comment les territoires s'adaptent aux bouleversements induits par la mondialisation, la décentralisation, la concurrence avec les voisins, mais aussi les mutations économiques et la mobilité grandissante des individus (Herbourg et alii, 2000). Ces phénomènes ont conduit à la désuétude des périmètres territoriaux hérités que sont la commune ou le département (Négrier, 2005).

18 Les territoires, et plus spécifiquement les territoires urbains, sont amenés à se rapprocher et à créer des gouvernances hybrides qui dépassent l'échelle des municipalités et des agglomérations. Cette recomposition territoriale a pris corps dans les années 1960 avec par exemple la création des districts urbains ${ }^{5}$. Mais c'est dans la décennie 1990 que la recomposition à l'échelle des villes fut la plus vive avec les lois permettant la création des communautés urbaines ou encore avec la politique des réseaux de villes portée par la DATAR.

La recomposition territoriale est un concept défini comme une «démarche institutionnelle entreprise par plusieurs collectivités territoriales pour réaliser un 
regroupement de leurs espaces de compétences» (Rolland-May, 2000A). Dans une acception plus fine, le concept englobe également la volonté de mise en commun des compétences et des ressources «par la lente maturation d'un sentiment collectif, par l'émergence d'une identité commune, par la volonté (et la capacité) de gérer conjointement les affaires quotidiennes et les projets" (ibid.). Cette volonté s'inscrit dans une temporalité relativement longue en comparaison avec le temps politique de plus en plus contracté. Les territoires les plus durables et les plus résilients sont ceux forgés de manière progressive.

Pourtant, la dynamique actuelle de constitution de nouveaux territoires politiques va dans l'ensemble à l'encontre de cette donne. En effet, nombreux sont les territoires à être créés à l'occasion de réformes législatives ou de manière opportuniste.

En 2000, Christiane Rolland-May a schématisé cette recomposition territoriale (Rolland-May, 2000A et 2000B). Elle l'avait d'ailleurs appliqué à l'« espace médiolorrain ", c'est-à-dire le bassin de vie de Pont-à-Mousson. Nous reprenons cette réflexion en l'étendant à l'ensemble de l'espace du Sillon Lorrain (figure 1). En effet, cette problématique de la recomposition territoriale n'exclut pas les espaces en réseau. La figure suivante, adaptation des schémas tirés des travaux de Rolland-May (ibid.), fait office d'élément de compréhension de la problématique soulevée ici.

Figure 1 : les trois étapes de la recomposition territoriale d'après Rolland-May

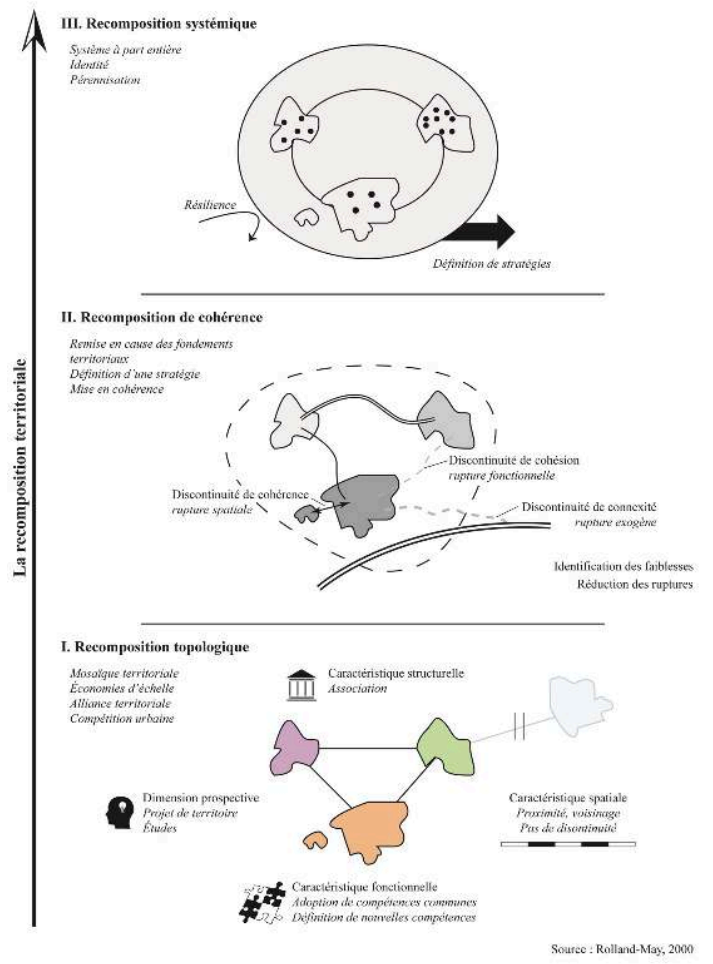




\section{De la métropole d'équilibre au pôle métropolitain : le Sillon Lorrain, une réalité déjà ancienne}

L'émergence des villes comme des acteurs à part entière se situe dans le prolongement de la régionalisation et s'accroît actuellement avec le passage à l'Acte III de la décentralisation. Si l'État fut un acteur central dans la réalisation de la décentralisation, les forces locales jouèrent également un rôle, tant dans leur degré de volontarisme que par les forces de proposition exprimées. Il s'agit donc d'un double mouvement. Le premier est un mouvement descendant issu de la volonté de l'État: donner progressivement aux régions les moyens d'un développement économique endogène. L'autre mouvement est ascendant. Il est né avec l'émergence des consciences régionales et l'existence de communautés d'intérêts tissées à cette échelle.

\subsection{La « métropole lorraine » : de la lente affirmation à l'échec}

Le Sillon Lorrain est une idée relativement récente. Elle a émergé à la fin des années 1990. Mais elle puise dans des racines plus anciennes. À partir des années 1950, dans un vaste élan décentralisateur, les régions sont progressivement mises en place en France. Parallèlement, les villes s'imposent progressivement comme des acteurs majeurs dans la volonté de décentraliser le pays et de faire émerger des pôles de croissance en Province. En Lorraine, les conséquences de cette ambition ont eu des répercussions sur la politique métropolitaine.

Avant de montrer les liens de filiation entre la métropole lorraine et le Sillon Lorrain, il convient de revenir sur le double mouvement de régionalisation/régionalisme qui a permis à la fois la naissance des régions - et plus particulièrement de la région Lorraine - en tant qu'institutions mais aussi comme des territoires à part entière.

\section{La naissance des institutions régionales et de l'opposition Metz-Nancy}

La rivalité Nancy-Metz est une réalité ancienne. Si la proximité géographique des deux villes explique en partie cette concurrence historique, les ruptures intervenues depuis le rattachement de la Lorraine à la France à la fin du XVIII ${ }^{e}$ siècle ont scellé cette opposition nord-sud. Cela obligea le pouvoir étatique à trancher systématiquement en faveur de l'une ou l'autre ville dans ses choix. Ainsi, tout au long du XIX ${ }^{\mathrm{e}}$ siècle, une série de décisions renforça la compétition entre les deux villes, le plus souvent à l'avantage de Nancy (Bonnefont, 1984 : 14) : construction du canal de la Marne-au-Rhin (inauguré en 1853), aménagement de la voie ferrée Paris-Strasbourg (mise en service en 1852 à Nancy).

Les tensions et les conflits franco-allemands ont également fortement pénalisé Metz. L'armée fut en effet le principal acteur de l'aménagement de part et d'autre de la nouvelle frontière établie par le traité de Francfort, imposant ses servitudes militaires. Des deux villes, Metz fut la plus touchée par les fortifications. L'annexion d'une grande partie de la Moselle par l'Allemagne à la suite de la défaite française renforça donc la dualité lorraine et accru encore la militarisation de la région et les servitudes défensives (Roth, 2011). Metz perdit plusieurs milliers d'habitants, particulièrement dans les classes aisées, et le retour à la France fut marqué par le départ des troupes allemandes (Arnould et Saint-Dizier, in Frécaut (dir.), 1983: 142). En accueillant 
notamment les Mosellans et les Alsaciens ayant opté pour la France, Nancy profita de l'annexion. Le renforcement de ses fonctions universitaire (avec le déplacement des facultés de pharmacie et de médecine de Strasbourg) et militaire ainsi que le développement de son économie grâce aux industriels ayant redéployé leurs entreprises du côté français ont fait de Nancy la nouvelle «capitale » de l'Est. Nancy s'imposa alors comme une ville industrielle.

Ainsi, dès les années 1950, alors qu'émerge un embryon de région Lorraine, la question métropolitaine prend forme en faveur de Nancy (Dion in Frécaut (dir.), 1983 : 346). En effet, la sélection des métropoles d'équilibre issue du rapport du commissariat général du Plan avait consacré la primauté métropolitaine lorraine à Nancy (Hautreux et alii, 1963). Pourtant, dans la décennie suivante, c'est bien une métropole d'équilibre multipolaire qui fut instaurée par la DATAR, parallèlement à la montée en puissance de Metz comme future capitale régionale, scellant ainsi la réalité polycentrique de la région et renforçant la difficulté du dialogue pourtant indispensable entre les deux villes.

En effet, deux éléments ont conduit les décideurs de l'État et les élus mosellans à renforcer Metz aux dépends de Nancy: d'une part, la volonté de compenser les handicaps messins résultants de l'annexion; d'autre part, le développement économique et démographique plus rapide du nord de la Lorraine. Ainsi, Metz pu récupérer le siège de la région militaire en 1946 alors qu'il fut initialement transféré à Nancy à l'issue de la guerre. Ceci préfigura l'obtention de la préfecture de région, puisque « la vocation des villes de résidence des préfets coordonnateurs à être chef-lieu de région fut enfin confirmée par une circulaire du 24 novembre 1961 [...] 》 (Masson, 1982: 498). Puis, en 1964, la CODER (Commission de Développement Économique Régional, ancêtre du Conseil Économique, Social et Environnemental Régional) fut installée à Metz. À la fin des années 1960, cette série de décisions provoquèrent une vive contestation des élus nancéiens qui transforma la simple méfiance entre Nancy et Metz en une véritable opposition, elle-même alimentée par les élus, les quotidiens locaux et l'opinion publique (ibid. : 503).

Le point d'orgue de la querelle entre les deux villes fut atteint en 1969 avec deux événements: d'une part, la révélation du choix d'installer définitivement le siège régional à Metz; d'autre part, le contentieux sur le tracé de l'autoroute ParisStrasbourg dont le choix du Ministère, alors occupé par Raymond Mondon, ancien maire de Metz, fut d'opter pour l'option nord afin de désenclaver l'agglomération messine. Malgré le maintien ou l'installation à Nancy de sièges administratifs, notamment de la nouvelle académie, et le renforcement de sa vocation universitaire, la fragile unité régionale était ainsi ébranlée (Dion, op. cit. : 347).

\section{La lente affirmation de la « métropole lorraine » avant sa mise en sommeil}

C'est dans ce contexte conflictuel que le gouvernement français décida de la création de la métropole d'équilibre Nancy-Metz-Thionville en 1966. Les métropoles d'équilibre avaient un double objectif: "faire contrepoids à Paris; donner une impulsion énergique au développement des régions qu'elles irriguent " (ORÉAM Lorraine, 1970). L'ambition de cette politique était forte, car il s'agissait de s'inscrire dans le temps afin de permettre le «renversement d'une tendance multiséculaire » (CITÉÉ, 1968: 4) : le monopole parisien et la macrocéphalie qui en découle (figure 2). 
Le choix d'associer à Nancy le couple Metz-Thionville tenait dans le faible rayonnement nancéien, comparativement aux autres métropoles d'équilibre françaises, et dans la volonté d'insérer la future région dans l'Europe des Six. Ainsi, la proximité frontalière de Metz et Thionville, au centre d'un triangle formé par Luxembourg, Sarrebruck et Nancy, fut jugée comme un atout pour la métropole. Très tôt, le principe du rôle moteur d'une métropole régionale sur son hinterland fut attendu et mis en avant. À l'époque, l'idée était de ne surtout pas reproduire la macrocéphalie nationale au niveau régionale («"la métropole et le désert lorrain" est une formule qui hante beaucoup»; ibid. : 5).

Figure 2 : les métropoles d'équilibre et leur zone d'influence

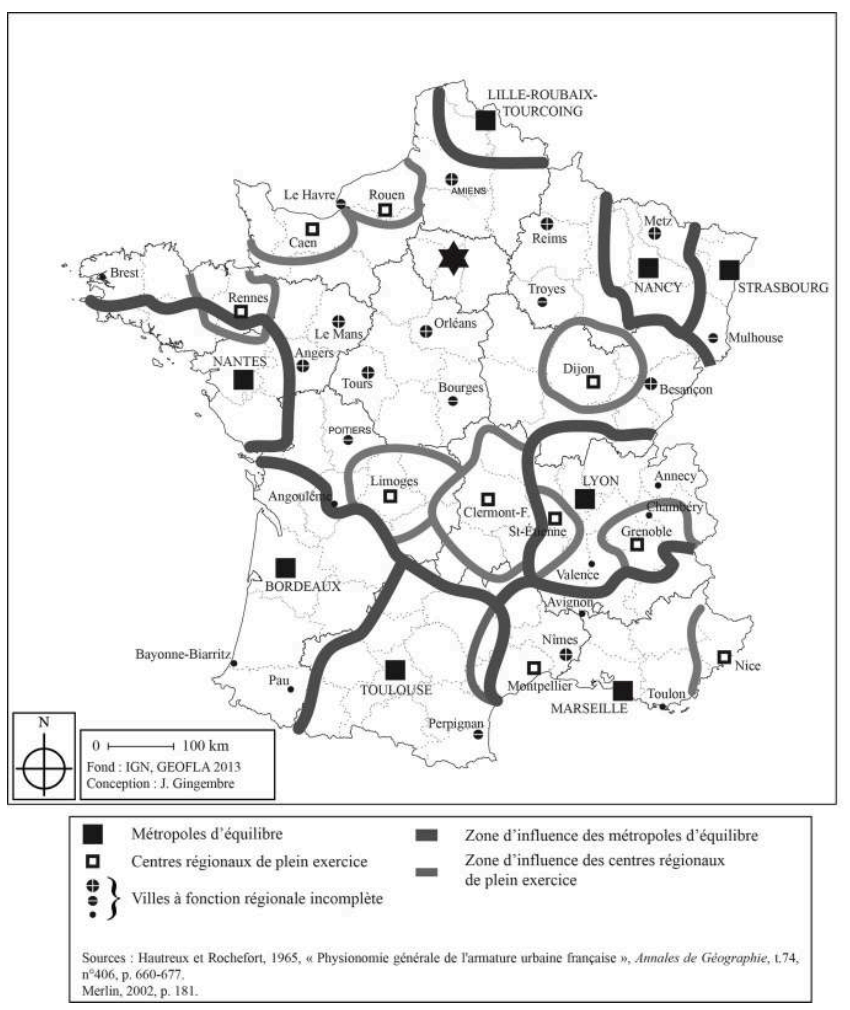

Crédit : Julien Gingembre, 2017

La métropole fut accompagnée de l'installation de l'ORÉAM à Pont-à-Mousson, localisation choisie afin de ne pas froisser Metz ou Nancy. Son rôle était triple : définir des objectifs de développement à long terme de la métropole; donner les moyens de la réalisation de ces objectifs par des principes d'aménagement déterminés ; proposer des actions à réaliser en conformité avec les orientations retenues (ORÉAM Lorraine, 1970 : 3-4). Avant de se lancer dans un programme d'aménagement de grandes infrastructures, les décideurs souhaitèrent en premier lieu passer par une phase de diagnostic et d'explication d'objectifs. Un Livre blanc fut ainsi rédigé afin de formuler ces objectifs en matière d'emploi, de services et d'aménagement (fonctions supérieures, transports, renforcement des principaux pôles urbains). Dans une perspective très aménagiste, cet ouvrage préconisait la réalisation de grands équipements métropolitains et l'ouverture européenne. Cette volonté de diagnostic et cette dimension prospective s'inscrivent dans la première étape de la recomposition territoriale, celle de la recomposition topologique. D'après Rolland-May (2000A), cette 
première étape repose sur quatre caractéristiques majeures : regroupement spatial d'entités proches (Nancy, Metz et Thionville); création de structures institutionnelles communes (ici, la métropole d'équilibre); adoption de compétences communes (à l'origine, il s'agit de la planification; l'aménagement vint ensuite); la dimension prospective.

La première action confiée à l'ORÉAM a donc consisté à établir le schéma d'aménagement de l'espace métropolitain. Son périmètre d'exercice réunissait 511 communes de Moselle et de Meurthe-et-Moselle et plus d'un million d'habitants. Les spéculations démographiques de l'époque imaginaient la croissance de la population des grandes agglomérations se poursuivre inexorablement jusqu'à la fin du siècle. Ces prévisions envisageaient que la Lorraine atteindrait 2700000 habitants en 1985. La réalité fut tout autre, puisqu'au recensement de la population de 1982, la Lorraine comptait 2319000 habitants (INSEE). Face à ces spéculations démographiques, le schéma d'aménagement devait envisager la création à l'horizon du milieu des années 1980 de nouveaux équipements métropolitains en matière de transports, d'enseignement supérieur, de tourisme ou encore de loisirs. Les propositions issues de l'analyse de l'ORÉAM préconisaient également une métropole étendue aux villes frontalières de Luxembourg et de Sarrebruck (ORÉAM Lorraine, 1970). Le diagnostic initial avait naturellement identifié la division de l'aire en deux secteurs principaux : l'agglomération de NancyToul-Lunéville au sud; les agglomérations de Metz et Thionville associées aux vallées sidérurgiques de la Fensch et de l'Orne au nord.

En 1973, l'Établissement Public foncier de la Métropole Lorraine (aujourd'hui EPFL) a été créé et marqua la seconde étape dans l'aménagement métropolitain de la région, celle de la maîtrise foncière (Jung, 1995).

Les choix en matière d'équipement du territoire contribuèrent à donner du sens à la "métropole lorraine» et plus généralement à l'esprit régional. Les transports constituaient un thème central dans le schéma directeur. L'autoroute A31 fut inaugurée dans les années 1960, tandis que le Métrolor fut lancé en 1970, avec un succès conséquent. Première desserte ferroviaire cadencée de France (hors région parisienne), elle permit de concrétiser fonctionnellement la métropole en reliant Nancy, Metz et Thionville. Enfin, la mise à grand gabarit de la Moselle débuta en 1964 et se poursuivit dans les années 1970 pour s'arrêter en amont au niveau du site sidérurgique de NeuvesMaisons.

Dans le même temps, le parc naturel régional de Lorraine vit le jour en 1974, et le projet de créer un lac artificiel pour les besoins de la ville de Metz évolua vers la réalisation d'une base de loisirs : le lac de Madine, dont les travaux furent terminés en 1978 (Jung, 1995). On cherchait aussi à répartir et renforcer équitablement les fonctions métropolitaines au sein des deux villes. Ces actions en faveur du renforcement de la métropole lorraine ont eu aussi vocation à installer durablement la région dans la partie qui se joue dans le grand espace de prospérité que constitue alors le couloir rhénan, dont les grandes villes proches (Strasbourg et Sarrebruck) constituent des pôles d'attractivité influents sur la région.

Ainsi, la métropole d'équilibre, par son ambition et des moyens mis en œuvre, a permis d'atteindre la seconde phase, la recomposition de cohérence. Cette étape est celle de l'aménagement et de la recherche d'une cohérence territoriale. Les actions pilotées par l'ORÉAM ont permis de réduire fortement les discontinuités spatiales, par des efforts orientés sur les problématiques des infrastructures de transport. Le Métrolor et le 
tronçon Metz-Nancy de l'A31 furent mis en œuvre au début des années 1970. Pendant que les discontinuités de cohésion interne étaient progressivement réduites, les discontinuités de connexité avec l'extérieur l'étaient également: ouverture de la Moselle canalisée à la navigation en 1964 permettant de rejoindre les grands ports de la mer du Nord, construction de l'A4 dans les années 1970. Toutefois, cette seconde phase demeura incomplète car coupée dans son élan par la remise en cause du concept de métropole multipolaire par la rivalité entre Metz et Nancy. La violente crise de l'industrie qui frappait la région a certainement exacerbé ces tensions.

Puis, la priorité donnée par l'action régionale au développement de l'aire métropolitaine durant le $\mathrm{VI}^{\mathrm{e}}$ Plan a conduit les institutions régionales à opérer un rééquilibrage avec le Plan suivant (1976-1980). Cela se fit notamment en orientant les efforts sur l'ensemble du territoire et particulièrement sur les villes petites et moyennes (Dion in Frécaut (dir.), 1983 : 348). Progressivement, durant la seconde moitié de la décennie 1970 et ce jusqu'aux années 1990, la question métropolitaine fut politiquement mise au second plan en Lorraine et en France. Cette « mise en sommeil » locale (Moriset, 1995: 60) coïncidait paradoxalement avec l'émergence du paradigme métropolitain dans la recherche française à partir du milieu des années 1980.

\subsection{Le Sillon Lorrain : un espace dans la fourchette base de sa recomposition territoriale}

39 L'émergence du concept de "métropolisation » à la fin des années 1980 a eu pour conséquence de remettre progressivement la question métropolitaine au centre du débat politique. Mais, contrairement aux métropoles d'équilibre des années 1960, la «métropole» des années 1990 n'est plus considérée comme un outil politique de l'aménagement volontariste de l'État. C'est désormais à la fois l'objet et le sujet de la métropolisation, concept qui se situe au centre d'un nouveau paradigme de recherche (Moriset, 1995 : 29 ; Ghorra-Gobin, 2010).

40 C'est dans ce contexte d'émulation autour de la question métropolitaine que Nancy et Metz se sont à nouveau rapprochées. Dès la fin des années 1980, les maires des deux villes évoquaient la structuration d'une nouvelle « métropole lorraine » (Moriset, 1997). Le CPER de seconde génération (1989-1993) consacra la notion de métropole bipolaire : il contenait un programme d'aménagement concerté du territoire spécifique à la métropole. Car le constat de l'époque était bien différent de celui des années 1960 : Nancy n'était plus considérée comme une métropole, c'est-à-dire une ville suffisamment influente pour rayonner sur toute la région et au-delà, et Metz n'avait pas non plus les atouts pour y prétendre, d'autant que la crise de la sidérurgie avait profondément touché le territoire lorrain.

41 Il était donc essentiel de rapprocher politiquement les deux villes, à défaut de les rapprocher spatialement. En effet, jugée comme handicapante dans les années 1960-1970, la rupture spatiale entre Nancy et Metz devait pouvoir être dépassée avec les nouveaux moyens de communication et la modernisation des transports réalisés dans les années 1990.

Ainsi, moins qu'une simple juxtaposition de deux pôles éloignés, le discours de l'époque misait beaucoup sur la figure structuraliste de l'axe métropolitain (Moriset, 1995 : 137). Cette anisotropie ${ }^{6}$ fut ainsi associée à la notion de "sillon mosellan " pour désigner la "métropole lorraine ». La figure du réseau devenait alors centrale dans la construction 
métropolitaine en Lorraine. Pourtant, la délimitation précise de l'aire métropolitaine restait lettre morte, impliquant ainsi une faiblesse d'un point de vue opérationnel.

Progressivement, l'idée d'une métropole linéaire s'étirant de la frontière luxembourgeoise (ou parfois même de Luxembourg) à Épinal faisait son chemin. Cette extension spatiale reposait certes sur des considérations économiques et démographiques. Mais elle était également politique. Au nord de la région, l'émergence du pôle luxembourgeois associée à l'ouverture des frontières imposèrent d'imaginer une métropole transfrontalière. Au sud, avec l'émergence du concept de «sillon mosellan ", se posait l'idée d'intégrer Épinal à l'ensemble, et ce pour deux raisons. D'abord, la mise à deux fois deux voies de la RN 57 entre Nancy et Épinal en 1991 rapprochait la préfecture vosgienne des espaces dynamiques situés au nord. Ensuite, face aux perspectives d'extension de la métropole vers le nord de la région envisagées par les élus de Metz, Nancy tendait la main à Épinal afin de ne pas se trouver en position de cul-de-sac et également pour renforcer son hinterland sud-lorrain (ibid. : 129).

L'idée d'un réseau de villes Thionville-Metz-Nancy-Épinal émergeait alors au tout début des années 1990. L'un des objectifs de ce rapprochement était de participer à la négociation du futur CPER 1994-1998. L'intégration d'un «contrat métropolitain » au Plan entérinait la reconnaissance obtenue dans le CPER précédent.

Alors que les réseaux de villes naissaient en France sur une initiative de la DATAR (Tesson, 1997), Nancy et Metz demeuraient absentes de cette stratégie. La priorité était plutôt mise dans la contractualisation avec l'État et la Région. Au début des années 1990, la rivalité entre Nancy et Metz demeurait encore trop accentuée pour permettre l'émergence d'une volonté politique forte (ibid.: 245). Cette frilosité s'est progressivement effacée durant la décennie, débouchant en 1998 sur une première réunion des élus de Thionville, Metz et Nancy. L'année suivante, le Sillon Lorrain est fondé par l'intermédiaire d'une déclaration commune avec le Conseil Régional. À l'aube $\mathrm{du} \mathrm{XXI}^{\mathrm{e}}$ siècle, cet acte fondateur lança une dynamique de structuration politique encore vive aujourd'hui.

L'étape majeure de la structuration du Sillon Lorrain fut la réponse à l'appel à coopération métropolitaine de la DATAR en 2004 (figure 3). Dans la foulée, en 2005, le Sillon Lorrain se structurait en association et se vit récompensé par la DATAR. Ceci améliora la visibilité du Sillon Lorrain et renforça sa cohésion politique interne. La création de l'association permit de capter les financements, de préparer des appels d'offre et d'organiser des rencontres prenant la forme de conférences ouvertes aux acteurs socio-économiques.

47 En 2007, le Sillon Lorrain cosignait le CPER de cinquième génération (2007-2013), assurant ainsi la continuité de la reconnaissance de la métropole dans le Plan depuis 1989. 
Figure 3 : les projets candidats et les lauréats de l'Appel à coopération métropolitaine de la DATAR en 2005

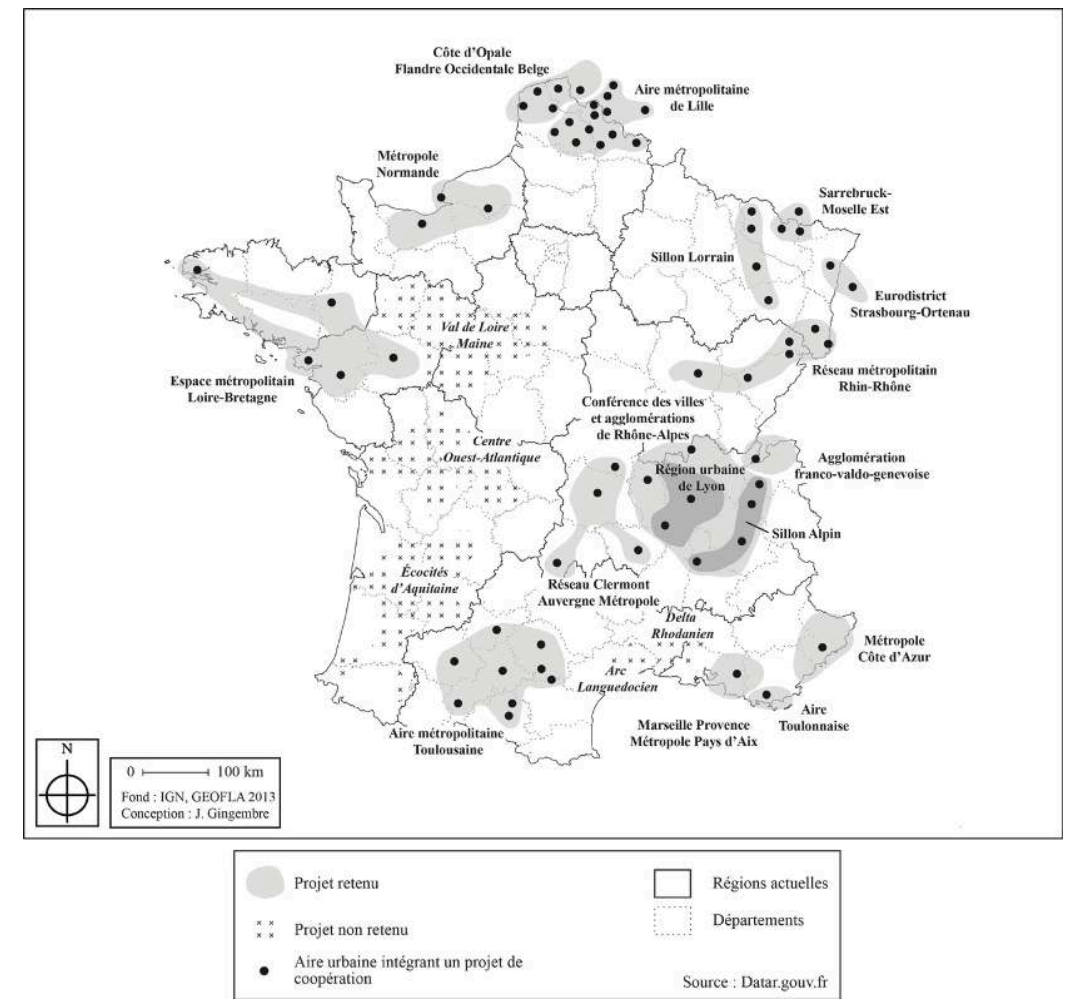

Crédit : Julien Gingembre, 2017

Pourtant, cet important travail de rapprochement ne fut pas de suite pleinement reconnu par l'État. En effet, le «rapport Balladur» (Comité pour la réforme des collectivités locales, 2009), dont le but était de proposer des mesures de simplification des collectivités territoriales françaises, préconisait la création de onze métropoles ${ }^{7}$ dont étaient notamment exclues Nancy et Metz. Ce choix fut mal vécu par les élus du Sillon Lorrain". Associés à d'autres élus de villes «oubliées » dans le rapport, les élus lorrains se mobilisèrent par la suite auprès du gouvernement et de la présidence de la République. Mené par André Rossinot, à l'époque maire de Nancy et actuel président de la métropole, l'important lobbying pour faire reconnaître la réalité métropolitaine multipolaire de la Lorraine aboutit à l'intégration dans la future Réforme des collectivités territoriales de 2010 d'un volet " pôle métropolitain » (Béhar et alii, 2011).

En février 2011, lors d'une réunion publique à Épinal, la décision de créer un pôle métropolitain fut prise. Le 18 décembre de la même année, le pôle métropolitain du Sillon Lorrain fut créé par arrêté préfectoral. Ceci permit au Sillon Lorrain d'être le premier pôle créé en France (figure 4). 
Figure 4 : les pôles métropolitains actuels et projetés

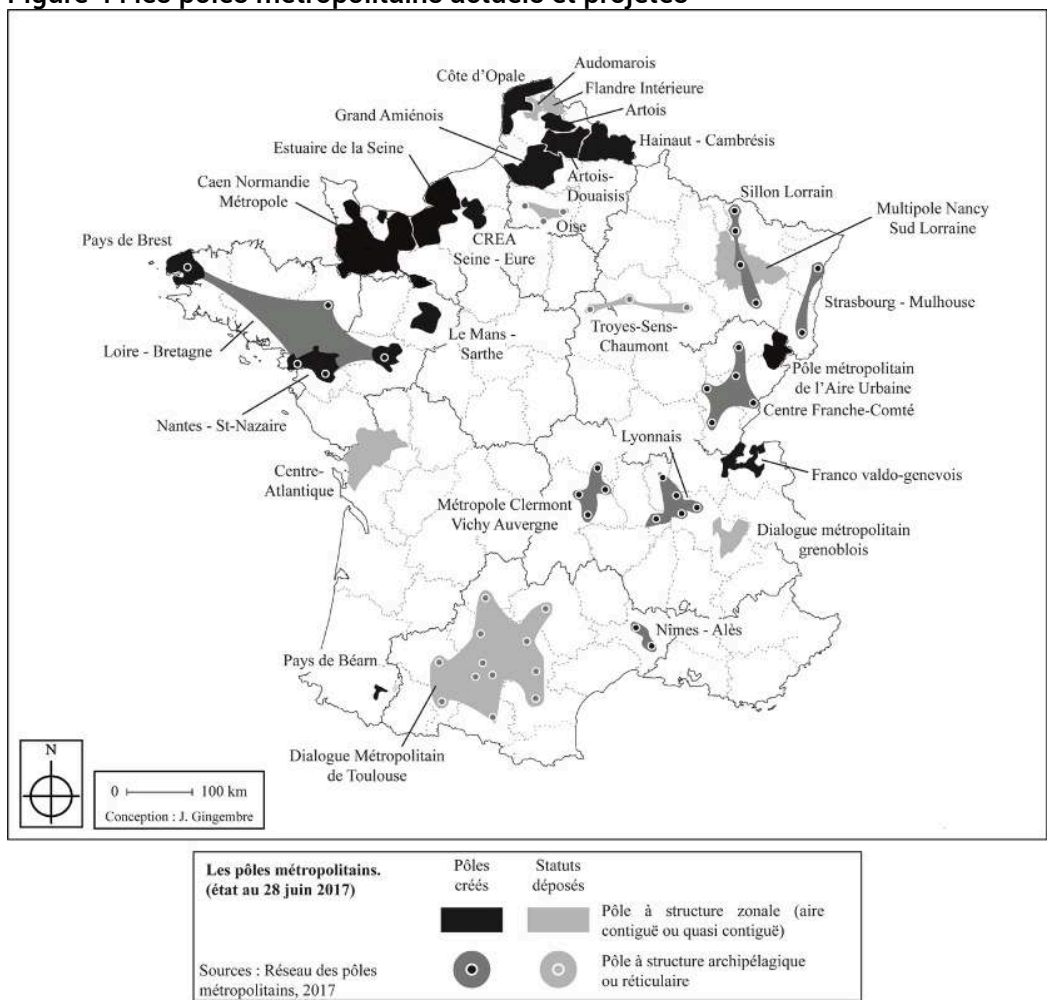

Crédit : Julien Gingembre, 2018 été transformée en «Conférence métropolitaine » (figure 5). Elle forme un organe à part entière du pôle depuis 2014 et se réunit régulièrement pour offrir une tribune aux élus du pôle. Elle leur permet de s'exprimer et de faire s'exprimer des chercheurs, des partenaires institutionnels, des personnalités de la société civile sur diverses thématiques (économie, mobilité, transfrontalier). Elle constitue également une sorte de «sas » pour les territoires qui n'intègrent pas le pôle mais qui souhaitent participer à ses réflexions et en devenir partenaire. C'est notamment le cas de Pont-à-Mousson ou de Saint-Dié-des-Vosges.

Parmi les premières actions menées par le pôle métropolitain du Sillon Lorrain figure la création d'une bibliothèque numérique de référence. Elle sera destinée à mettre en commun et en ligne le patrimoine littéraire, scientifique et documentaire des bibliothèques des quatre villes ${ }^{9}$. L'autre projet majeur porté par le pôle est LORnTECH. Il s'agit d'une fédération qui regroupe des entreprises (généralement des start up, des laboratoires de recherches, des écoles, des collectivités) autour des technologies du numérique. Sorte de technopôle "déterritorialisé ", LORnTECH s'appuie sur la mise en réseaux de ces acteurs. Son activité a été reconnue par l'obtention du label FrenchTech décerné à de telles initiatives par le Ministère de l'Économie en 2014 et en 2015. Cette labellisation comprend un financement des activités et a pour ambition de promouvoir l'économie numérique française à l'étranger. Au-delà de l'intérêt de la fédération d'acteurs, l'objectif est de faire émerger au sein de ce réseau des entreprises à fort potentiel de développement économique.

Ces réalisations demeurent toutefois relativement abstraites. Elles ne sont que des ébauches qui préfigurent des projets plus consistants. Mais la difficulté tient dans 
l'absence de compétences réelles du pôle métropolitain, malgré l'ambition de la loi pour qui les pôles promeuvent des "actions d'intérêt métropolitain en matière de développement économique, de promotion de l'innovation, de la recherche, de l'enseignement supérieur et de la culture, ou d'aménagement de l'espace » (Code général des collectivités territoriales, art. L5731-1). Le pôle s'apparente ainsi plutôt à un organe de dialogue et d'influence auprès des partenaires, des collectivités et de l'État. L'autre limite du pôle se situe de son incapacité à fédérer les acteurs lorsque des points de mésentente émergent au sein du Sillon Lorrain.

Figure 5 : la démarche institutionnelle ayant mené à la création du pôle et fonctionnement de la gouvernance du Sillon

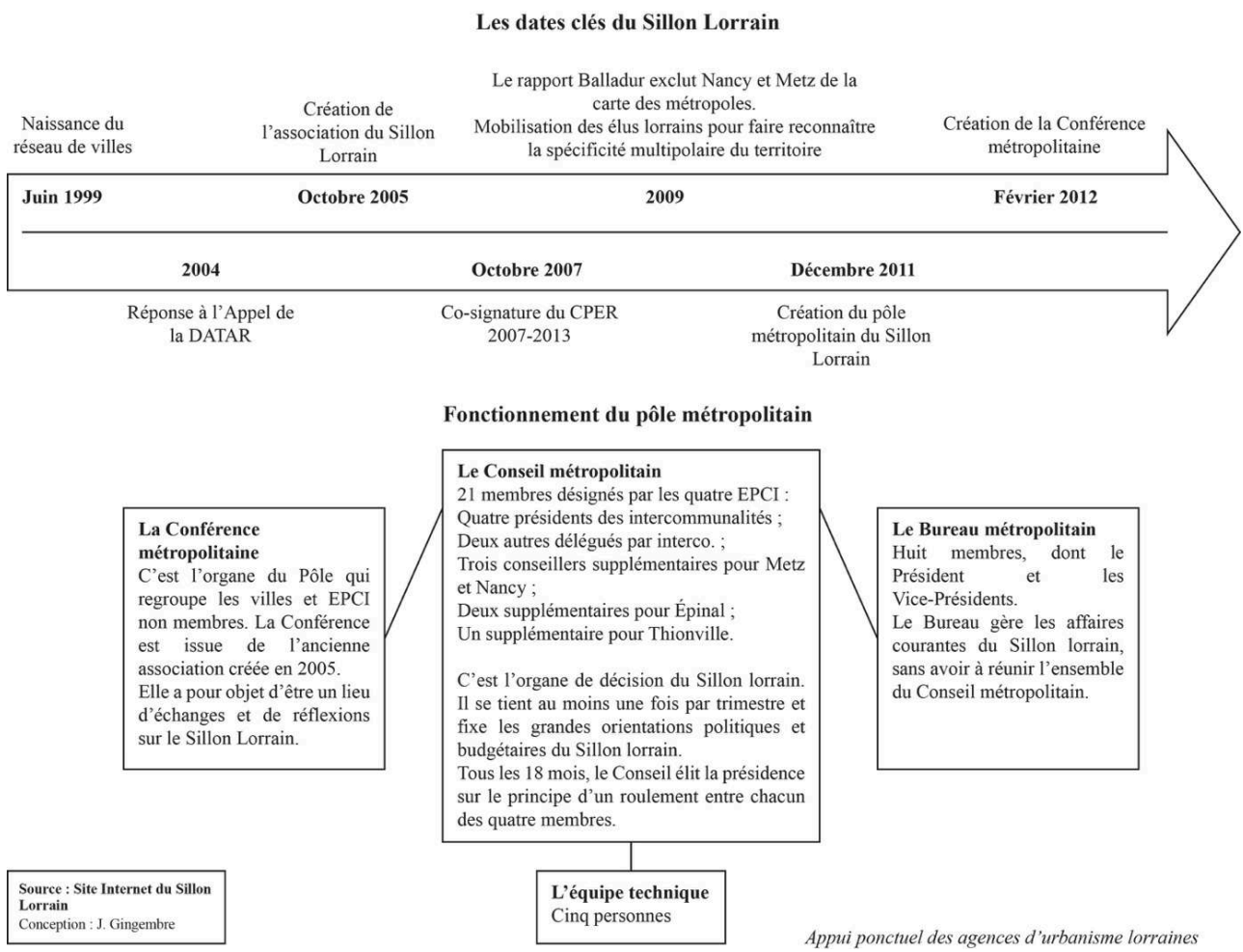

Crédit : Julien Gingembre, 2018

\subsection{Le maintien des blocages internes}

En Lorraine, l'un des points de discorde emblématiques des années 2000 en matière d'aménagement est la question de l'implantation d'une gare d'interconnexion TGV-TER. En effet, lors de l'ouverture de la LGV Est en 2007, la question de la centralisation dans une seule et même gare des trajets pour Paris et les villes de Province a été posée. Afin de ne pas raviver la tension Nancy-Metz, une solution de compromis fut trouvée : les gares des deux villes sont reliées directement à Paris ; pour se rendre dans une autre ville desservie par le TGV, il faut prendre le train dans une gare située à mi-chemin entre Nancy et Metz. À l'instar de l'aéroport régional de Lorraine, installé en Moselle à $25 \mathrm{~km}$ au sud de Metz et à $35 \mathrm{~km}$ au nord de Nancy, la gare de Louvigny (Moselle) est implantée en rase campagne. Cette situation singulière est révélatrice des difficultés persistantes d'opérer des choix d'aménagement entre Metz et Nancy. 
54 À l'origine, plusieurs options furent retenues : installer une gare sur la ligne TER NancyMetz à Pagny-sur-Moselle ou à Vandières, à mi-distance des deux préfectures; ou construire une gare en rase campagne. Dans le premier cas, bien que Pagny-sur-Moselle est bien située et constitue un nœud ferroviaire régional, les mesures de protections environnementales de la vallée du Rupt-de-Mad ont empêché le projet. Le second cas impliquait quant à lui des dispositifs techniques complexes et coûteux (viaduc, gare sur deux niveaux). Face à ces difficultés et à l'absence de concorde dans le territoire, le choix s'est porté sur le troisième choix, malgré l'opposition de certains élus locaux, particulièrement les élus de gauche (Michel Dinet, président du conseil départemental de Meurthe-et-Moselle, et Jean-Pierre Masseret, président du conseil régional de Lorraine, en tête). Bien que la connexion TGV-TER était inscrite dans la déclaration d'utilité publique, la gare de Louvigny fut mise en service en 2007 pour l'arrivée du TGV, avec l'idée qu'elle ne serait que provisoire.

En 2014, la question de la gare d'interconnexion revient dans le débat politique régional, par l'intermédiaire du président de région Jean-Pierre Masseret, qui souhaite interroger les Lorrains par referendum. Malgré un avis défavorable de la cour régionale des comptes, l'État était prêt à participer au financement. En février 2015, la consultation, marquée par une très forte abstention (90\%), marque la victoire du «non ». Tel un serpent de mer, le projet n'est à l'heure actuelle pas complètement enterré par la région Grand Est et la SNCF.

Le comportement des acteurs politiques joue ici un rôle central. Les dissensions entre Nancy et Metz ne sont pas forcément la seule cause du blocage sur ce dossier: les divergences entre les départements de Meurthe-et-Moselle et de Moselle, et la région (Lorraine puis Grand Est) ont ici joué un rôle important.

57 Ces blocages empêchent le Sillon Lorrain de poursuivre sa recomposition. Pourtant, par son ancienneté et surtout par son origine puisée dans la démarche de la métropole d'équilibre lorraine, il s'inscrit dans la première étape de la recomposition territoriale (figure 1). En effet, la proximité géographique est à la base de la démarche qui est portée par les élus acteurs et les acteurs locaux. Cette étape se caractérise par le choix de créer une structure commune (ici, le pôle métropolitain) notamment pour réunir les financements. La délimitation d'une aire pertinente est donc centrale. L'échelle varie de l'agglomération au réseau de villes. Des compétences communes sont également transférées depuis les collectivités membres : pour le Sillon Lorrain, il s'agit d'une part d'« actions visant à défendre les intérêts et à renforcer l'identité du pôle », et, d'autre part, de «mettre en place une ingénierie pour le développement de projets et la captation de financements » (site Internet du pôle).

58 Cette première étape ne garantit donc pas la pérennité ou la cohérence de la structure. En effet, le pôle métropolitain doit démontrer son utilité dans le temps et s'inscrire dans un projet cohérent. Le blocage ici tient donc notamment dans le faible degré d'intégration du pôle. Nous l'avons vu, les compétences qui lui ont été transférées sont peu nombreuses et relèvent plutôt d'une logique d'impulsion et de mise en cohérence de projets. Cela se révèle peu structurant d'un point de vue spatial. Chacune des villes poursuit individuellement son développement, parfois à l'encontre de la cohérence globale. C'est par exemple le cas avec la réalisation à Nancy en 2014 d'un palais des congrès, le Centre Prouvé, tandis que Metz attend d'inaugurer le sien (le futur centre des congrès Robert Schuman). Une solution commune aurait pu être envisagée. À un 
autre niveau impliquant d'autres acteurs politiques, le cas de la gare d'interconnexion révèle également le manque de cohérence dans les prises de décision.

Dans la suite logique de la première étape, la seconde étape (recomposition de cohérence) consiste à identifier puis à réduire les ruptures et les discontinuités géographiques internes et externes à l'aire d'exercice de la structure. Il s'agit surtout d'actions relevant de l'aménagement du territoire, ce que le pôle n'est pas en mesure de réaliser directement. Mais il peut être à l'origine d'une mise en cohérence de projets. Surtout, il peut pleinement jouer son rôle d'incitateur auprès des nouvelles instances régionales et de l'État.

La troisième et dernière étape de la recomposition territoriale est la « recomposition systémique ». C'est par exemple le niveau atteint par la métropole du Grand Nancy, du fait de sa longue histoire et de sa capacité intégratrice dont elle fait preuve. Ces territoires font preuve de pérennité, d'invariance et même de résilience face aux changements, qu'ils soient lents ou brutaux. Pour cela, leurs compétences doivent être variées afin de pouvoir agir sur un maximum de composantes à la fois économiques, spatiales, sociales, environnementales et culturelles. La gouvernance y tient un rôle central, de même que la légitimité démocratique des décideurs et des réseaux d'acteurs en place.

61 Cette rétrospective illustre la difficile mise en place en France d'une politique métropolitaine. Ces difficultés furent exacerbées en Lorraine. La région constituait déjà un cas de figure unique qui a dès le départ embarrassé la DATAR. La concurrence entre Metz et Nancy, au lieu d'être dépassée par la mise en place de la métropole, a même été renforcée au point de mettre à mal la fragile unité. Les deux villes se tournèrent le dos durant près de deux décennies. C'est le nouveau contexte des années 1990 qui offrit l'occasion à Metz et Nancy de tenter un nouveau rapprochement, cette fois-ci sans le chaperonnage de l'État. Encore une fois, la Lorraine fit figure d'avant-gardiste en mettant sur pied à la fin des années 1990 un véritable réseau de villes. Durant toute l'existence de la métropole d'équilibre, les travaux et études de l'oréAm ont surtout porté sur les thématiques des transports et de la mobilité. Cette problématique a été à juste titre définie comme prioritaire afin de réduire la distance entre les deux villes principales. Le renouveau du dialogue lorrain porté par le Sillon depuis maintenant près d'un quart de siècle a remis cette thématique au centre des enjeux régionaux.

\section{Conclusion}

Le pôle métropolitain tente une gouvernance d'un espace à forts enjeux dans le cadre de la récente fusion régionale. Les logiques nord-sud y prédominent toujours, héritages de la géographie et de l'histoire, même si l'État, par l'intermédiaire de la LGV Est, et l'Europe, avec son RTE-T (Réseau Transeuropéen de Transport), s'efforcent de bouleverser cet état des choses. Deux corridors européens traversent ainsi le Sillon : ils sont constitués d'axes de transport (fluvial, ferré et routier) prioritaires pour l'Union européenne. La problématique des transports constitue l'un des questionnements les plus sensibles pour l'avenir du pôle. La carte suivante tente une synthèse de ces enjeux (figure 6). 
Figure 6. Les infrastructures de transport dans le Grand Est, état des lieux et perspectives

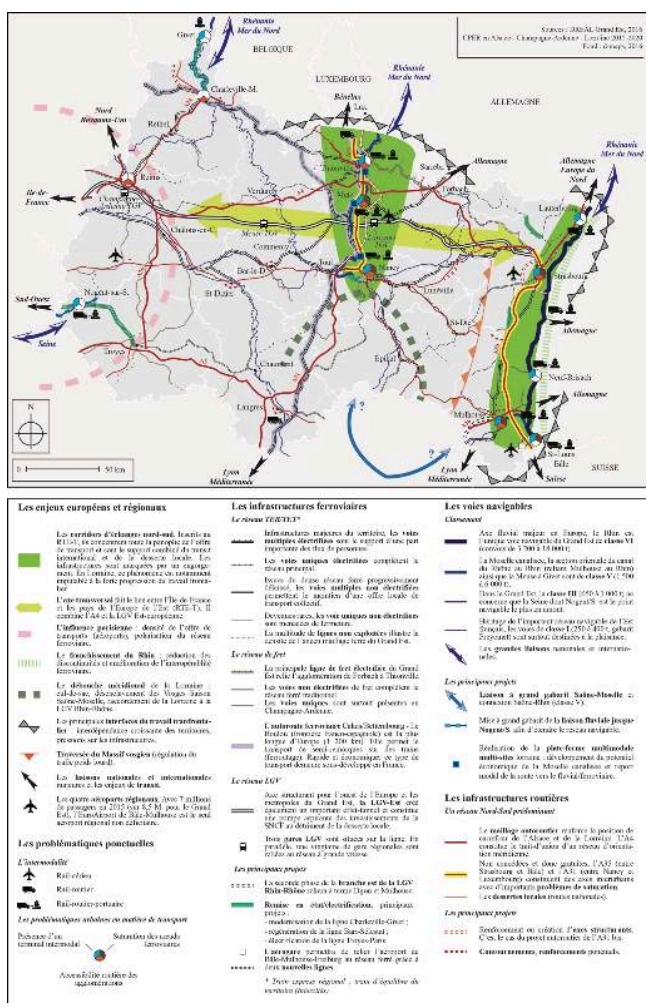

Crédit : Julien Gingembre, 2017

63 L'accessibilité du Sillon Lorrain revêt donc un caractère stratégique pour le développement futur des activités économiques. Dans un contexte de désinvestissement croissant de l'État dans les grands projets d'infrastructures, la volonté est aujourd'hui de renforcer ce qui existe. L'élaboration de nouveaux projets majeurs est rare, hormis la réalisation de l'A31 bis. Le projet de liaison Rhin-Rhône demeure aujourd'hui hypothétique. Mais malgré l'ambition affichée d'améliorer les transports dans cet espace carrefour et de désenclaver les espaces ruraux, force est de constater que la concentration des efforts sur les espaces principaux se fait au détriment des liaisons secondaires, notamment les voies ferrées. Les débats actuels sur l'avenir du rail en France laissent augurer une nouvelle déliquescence du réseau ferré secondaire.

Espace frontalier majeur, le Sillon Lorrain se tourne également de plus en plus vers ses voisins européens, en particulier en matière d'emploi (figure 7). Il s'agit de l'autre question épineuse posée sur l'avenir du pôle. Celui-ci est tiraillé entre plusieurs dynamiques, ce qui a des conséquences sur le plan interne. Metz et Thionville sont les meneurs de cette ambition transfrontalière. Nancy et Épinal demeurent quant à elles plutôt tournées vers d'autres stratégies (le Bassin parisien ou encore le couloir rhodanien), sans négliger la dynamique frontalière. 
Figure 7. Les flux de mobilité domicile-travail entre les agglomérations lorraines et vers l'étranger (2009), une illustration de la réalité fonctionnelle du Sillon Lorrain
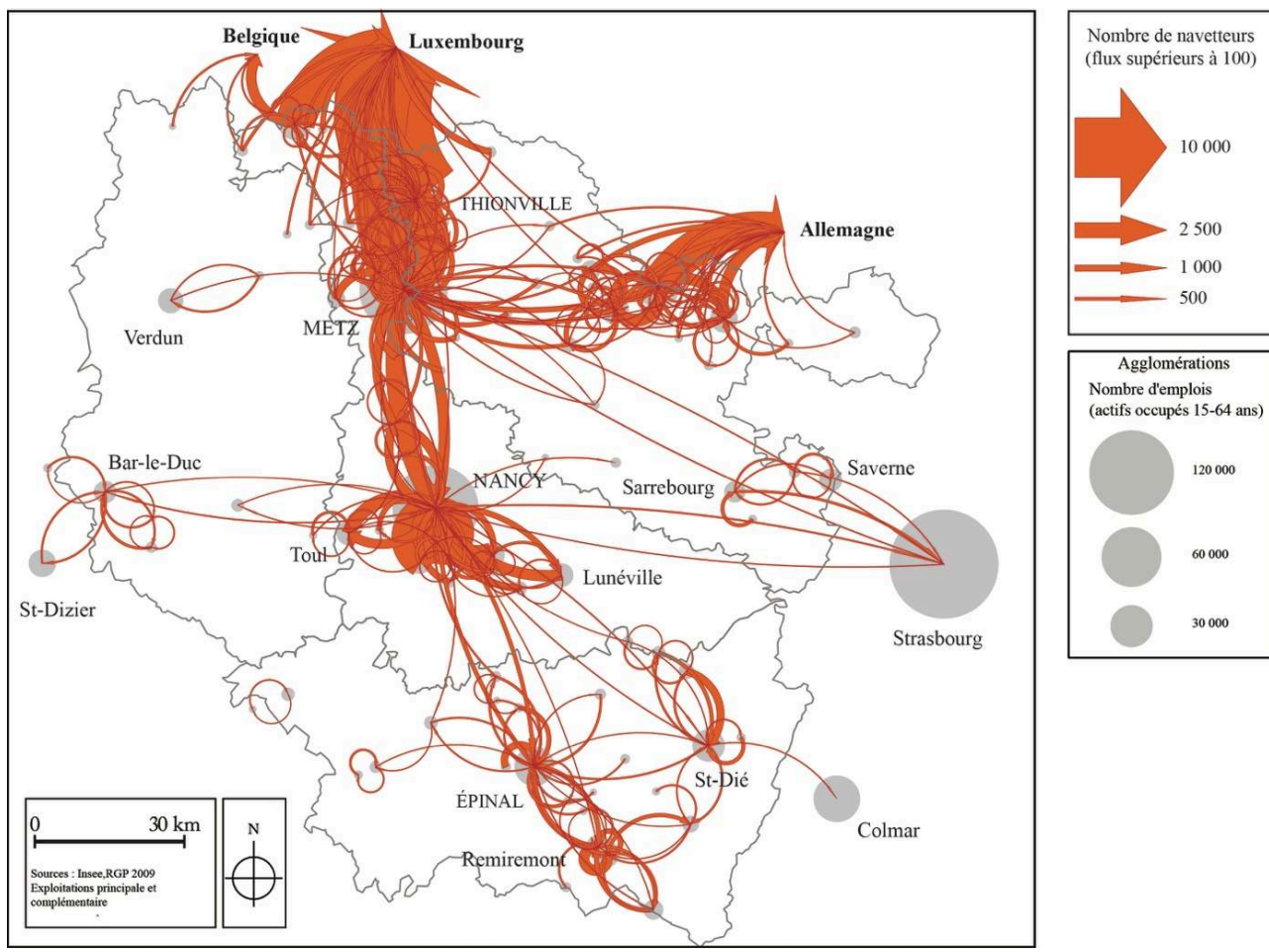

Agglomérations Nombre d'emplois (actifs occupés 15-64 ans)

Crédit : Julien Gingembre, 2017

La création du pôle métropolitain et de métropoles à Nancy et à Metz s'inscrit dans l'ambition d'un rayonnement des villes lorraines aux échelles nationales et européennes. Il s'agit également d'une volonté de se positionner sur la carte des métropoles, face à des voisins plus importants d'un point de vue démographique (Strasbourg) ou économique (Luxembourg). Contrairement à d'autres villes françaises, telles que Nantes où les indices économiques et démographiques sont positifs (Fritsch, 2006), Metz et Nancy souffrent de l'atonie du territoire lorrain. Mais elles ne sont pas dénuées d'ambitions. Les enjeux inhérents à cette recomposition territoriale se situent également sur un plan interne. Il s'agit en effet de poursuivre le développement du territoire et d'en améliorer la cohésion.

Pourtant, l'enjeu de la solidarité territoriale est central dans cet espace encore touché par des pertes d'emplois. De même, la concentration des richesses, des équipements et des populations les plus favorisées dans les principales villes est un phénomène de plus ne plus remis en cause actuellement (Dumay, 2018). Cela a notamment été observé dans le Sillon Lorrain à propos des catégories socio-professionnelles (Gingembre, 2016). Le Sillon doit ainsi démontrer son utilité comme rôle-moteur auprès des citoyens. Faute de quoi cette recomposition pourrait être mal comprise en s'apparentant à de nouvelles « couches » dans le « mille-feuille » territorial français.

La difficulté tient alors dans la conciliation entre les ambitions locales - celles de Nancy et de Metz devenues métropoles, et celle de Thionville et de Metz qui envisagent la fusion - et les ambitions régionales qui ne seront désormais plus portées à Metz mais à Strasbourg. Pour ne pas diluer les intérêts lorrains dans ce vaste ensemble régional, le Sillon Lorrain semble avoir ici une importante carte à jouer. 


\section{BIBLIOGRAPHIE}

Ascher F., 1995, Métapolis ou l'avenir des villes, Paris, Odile Jacob.

Béhar D., Estèbe P., Vanier M., 2011, « Pôles métropolitains : du faire territoire au faire politique », Métropolitiques, 18 mai 2011, en ligne (28 avril 2018).

http://www.metropolitiques.eu/Poles-metropolitains-du-faire.html

Bonnafous A., 1997, « Le projet de liaison Rhin-Rhône à gabarit européen ou les mystères d'un pari ", Annales de Géographie, vol. 106, n 593, pp. 107-128.

Bonnefont J.C., 1984, La Lorraine, Paris, Presses Universitaires de France.

Bozzani-Franc S., 2006, Grandes vitesses, métropolisation et organisation des territoires : l'apport de l'intermodalité aéro-ferroviaire à grande vitesse au rayonnement métropolitain, Thèse de doctorat, Lille. Brunet R. et Dolfus O., 1990, Géographie universelle, vol. 1 : Mondes nouveaux, Paris, Hachette.

Citee, 1968, « La métropole lorraine », Équipement, logement, transports, n 35.

Conseil régional d'Alsace, 2015, Contrat de Plan État-Région 2015-2020, Strasbourg, 99 p.

Conseil régional de Champagne-Ardenne, 2015, Contrat de plan État-Région 2015-2020 de la

Champagne-Ardenne, Châlons-en-Champagne, 128 p.

Conseil régional de Lorraine, 2015, Contrat de Plan État-Région Lorraine 2015-2020, Metz, 78 p.

Dumay J.-M., 2018, « La France abandonne ses villes moyennes », Le Monde diplomatique, mai 2018, pp. 22-23.

Dumont G.-F., 2006, L'attractivité des métropoles moyennes en France, Paris, DIACT.

Ehrlacher F. (dir.), 2015, Le transport en Lorraine : les chiffres-clés édition 2015, Metz, ORTL.

Facchinetti-Mannone V., Bavoux J.-J., 2010, «L'implantation des gares TGV en France : tensions interscalaires, jeux d'acteurs et recompositions spatiales », Belgeo, 1-2/2010, pp. 9-22.

Frécaut (dir.), 1983, Géographie de la Lorraine, Nancy, Presses Universitaires de Nancy.

Fritsch B., 2006, « Nantes - Saint-Nazaire : métropole exemplaire? », L'information géographique, 2006/4, vol. 70, pp. 25-45.

Ghorra-Gobin C., 2010, « De la métropolisation : un nouveau paradigme? », Quaderni, vol. 73, n 3, pp. 25-33.

Gingembre J., 2017, Vers des territoires métropolisés en réseau ? Le cas émergent du Sillon Lorrain, Thèse de doctorat, Nancy, $394 \mathrm{p}$.

Gingembre J., 2016, « La spécialisation résidentielle dans le Sillon Lorrain », Revue Géographique de l'Est, vol. 56 / n 3-4, en ligne (29 avril 2018).

http://journals.openedition.org/rge/5922

Gingembre J., Baude J., 2014, « Les mobilités domicile-travail dans les réseaux d'agglomérations », EchoGéo, 2014/27, en ligne (29 avril 2018).

Hautreux J., Lecourt R., Rochefort M., 1963, Le niveau supérieur de l'armature urbaine française, Paris, Commissariat général du Plan. 
Hecker A., 2015, « Les infrastructures de transport, vecteurs d'intégration métropolitaine ? Le cas du Sillon Lorrain », Géotransports, n 5-6, pp. 87-102.

Herbourg A.M., Lallement G., Loubignac P., 2000, « La recomposition des territoires : un facteur déterminant de l'action régionale ", Revue Géographique de l'Est, vol. 40/4, en ligne (29 avril 2018). https://journals.openedition.org/rge/4071

Jung J., 1995, « Souvenirs d'un aménageur lorrain », in : Académie nationale de Metz (sous la direction de), Mémoires de l'Académie nationale de Metz, Metz, pp. 9-30

Masson J.-L., 1982, Histoire administrative de la Lorraine : des provinces aux départements et à la région, Paris, Éditions F. Lanore.

Merlin P., 2002, L'aménagement du territoire, Paris, PUF.

Moriset B., 1995, Nancy-Metz : de la métropole d'équilibre à la métropolisation, discours et mythes, Thèse de doctorat, Grenoble.

Moriset B., 1997, «La délimitation des aires métropolitaines : représentations et enjeu politique, l'exemple de la métropole lorraine Nancy-Metz ", Annales de géographie, vol. 106, n 595, pp. 243-262.

Négrier E., 2005, La question métropolitaine : les politiques à l'épreuve du changement d'échelle territoriale, Grenoble, Presses Universitaires de Grenoble.

OREAM Lorraine, 1970, Schéma d'aménagement de la métropole lorraine, Pont-à-Mousson, OREAM.

Rey A., 2006, Dictionnaire historique de la langue française, Paris, Le Robert.

Rolland-May C., 2000A, « Géostratégie de la recomposition de territoires : cas particulier en espace fortement métropolisé, l'espace « médio-lorrain » autour de Pont-à-Mousson », Revue Géographique de l'Est, vol. 40/4, en ligne (29 avril 2014).

https://journals.openedition.org/rge/4015

Rolland-May C., 2000B, Évaluation des Territoires. Concepts, modèle, méthodes, Paris, HermèsSciences.

Roth F., 2011, La Lorraine annexée : étude sur la présidence de Lorraine dans l'Empire allemand 1870-1918, Metz, Éditions Serpenoise.

Santamaria F., 2012, « Les villes moyennes françaises et leur rôle en matière d'aménagement du territoire : vers de nouvelles perspectives ? ", Norois, 2012/223, pp. 13-30.

Selz G., 1968, « La métropole lorraine », Équipement, logement, transports, Centre d'information technique et économique de l'équipement, $\mathrm{n}^{\circ} 35$.

Siino C., Laumiere F., Leriche F. (dir.), 2004, Métropolisation et grands équipements structurants, Toulouse, Presses universitaires du Mirail, 305 p.

Tesson F., 1997, «Les expériences françaises de réseaux de villes : des dynamiques pour de nouveaux territoires ", Flux, 13, 27, p. 25-40.

Vanier M., 2010, Le pouvoir des territoires : essai sur l'interterritorialité, Paris, Économica : Anthropos.

Woessner R., 2006, « L'éternel retour du Corridor Rhin-Rhône », Annales de géographie, vol. 115, $\mathrm{n}^{\circ} 647$, pp. 2-25.

Woessner R., 2004, « Le Grand Est français : une région incomplète », Les Cahiers scientifiques du transport, n 46/2004, pp. 45-62. 


\section{NOTES}

1. Formules régulièrement utilisées par les élus du pôle, comme Dominique Gros, maire de Metz, dans le Républicain Lorrain, premier avril 2012.

2. Le Grand Est, qui réunit également l'Alsace et la Champagne-Ardenne depuis le 1er janvier 2016.

3. Par exemple le $n^{\circ} 378$ (mai - juin 2011) de la revue Urbanisme, «Les villes moyennes contreattaquent $»$.

4. Thionville et Épinal font partie des douze villes lorraines retenues.

5. Celui de Nancy a été créé en 1959.

6. L'isotropie caractérise les phénomènes «qui présentent les mêmes propriétés dans toutes les directions. » Son antonyme, l'anisotropie, désigne les phénomènes « qui possèdent des propriétés physiques variant selon la direction considérée » (Rey, 2006 : 1889).

7. Les communautés urbaines de Lyon, Lille, Marseille, Bordeaux, Toulouse, Nantes, Nice, Strasbourg, ainsi que les communautés d'agglomération de Rouen, Toulon et Rennes étaient proposées dans le rapport.

8. Le Journal des Entreprises, 2 octobre 2009.

9. Baptisée Limedia, le site a été lancé en avril 2018. Avec 100000 références, il sera enrichi au cours de l'année.

\section{RÉSUMÉS}

Fortes d'une expérience initiée dès les années 1960 avec la politique des métropoles d'équilibre, Nancy et Metz s'inscrivent désormais au sein du pôle métropolitain du Sillon Lorrain aux côtés de Thionville et d'Épinal. Cette réalité politique ancienne tente aujourd'hui une gouvernance complexe sur un espace encore peu intégré où les ambitions individuelles des quatre villes demeurent prégnantes. Pourtant, la recomposition territoriale en action s'appuie sur une réalité géographique et fonctionnelle. L'article montre toute la singularité de la construction métropolitaine en Lorraine, inscrite dans le temps long. Les nouvelles perspectives apportées par la récente réforme de la carte régionale renforcent les enjeux dans le Sillon Lorrain, espacecarrefour majeur entre la France et l'Europe rhénane.

Since the 1960s, Nancy and Metz forms a metropolitan cooperation area. Today, within the Metropolitan Pole of the Sillon Lorrain with Thionville and Épinal, cities are part of complex governance over a space that is still little integrated. Individual logics remain important. The "territorial recomposition" in action is based on a geographical and functional reality. The new prospects brought about by the recent reform of the regional map reinforce the stakes in the Sillon Lorrain, a major crossroads between France and Rhine Europe.

\section{INDEX}

Thèmes : Carnets de recherches

Mots-clés : Sillon Lorrain, recomposition territoriale, métropolisation, gouvernance

Keywords : Sillon Lorrain, Territorial Recomposition, Metropolisation, Governance 
AUTEUR

JULIEN GINGEMBRE

Docteur en géographie

Chercheur associé au laboratoire LOTERR

Université de Lorraine

julien.gingembre[at]univ-lorraine.fr 